\title{
Matching illumination of solid objects
}

\author{
Sylvia C. Pont And Jan J. Koenderink \\ Helmholtz Institute, Utrecht University, Utrecht, The Netherlands
}

\begin{abstract}
The appearance of objects is determined by their surface reflectance and roughness and by the light field. Conversely, human observers might derive properties of the light field from the appearance of objects. The inverse problem has no unique solution, so perceptual interactions between reflectance, roughness, and light field are to be expected. In two separate experiments, we tested whether observers are able to match the illumination of spheres under collimated illumination only (matching of illumination direction) and under more or less diffuse illumination (matching of illumination direction and directedness of the beam). We found that observers are quite able to match collimated illumination directions of two rendered Lambertian spheres. Matching of the collimated beam directions of a Lambertian sphere and that of a real object with arbitrary reflectance and roughness properties resulted in similar results for the azimuthal angle, but in higher variance for the polar angle. Translucent objects and a tennis ball were found to be systematic outliers. If the directedness of the beam was also varied, the direction settings showed larger variance for more diffuse illumination. The directedness settings showed an overall quite large variance and, interestingly, interacted with the polar angle settings. We discuss possible photometrical mechanisms behind these effects.
\end{abstract}

The appearance of an object is determined by the light field, the geometry of the object, its reflectance, and its roughness. Conversely, the appearance of objects is the only cue to the light field. This problem is underdetermined and suffers from ambiguities, such as the bas-relief ambiguity (Belhumeur, Kriegman, \& Yuille, 1999), the shapeshading ambiguity (Langer \& Bülthoff, 2001), and the illumination-reflectance ambiguity (Adelson \& Pentland, 1996; Dror, Willsky, \& Adelson, 2004). Without assumptions about the object geometry, reflectance, and light field, it would not be possible, for instance, to derive the illumination direction from the images in Figure 1. Using a same-different paradigm, we found that material and illumination perception are basically confounded in the cases of artificial smooth spheres, of real smooth spheres under natural illumination, and of real rough spherical objects under canonical illumination (te Pas \& Pont, 2005). However, human observers seem to be able to judge at least the lower order properties of the light field in a scene, such as the mean illumination direction and whether the illumination is more or less diffuse (less or more directed). Furthermore, we expect the higher order angular frequencies in the radiance distribution to be relevant for perception in a statistical sense. The light field itself cannot be observed, but its effect on the appearance of objects in a scene can. Therefore, one might expect that humans use the appearance of objects in scenes in order to estimate the light field. The aim of this study was to test whether observers are able to derive illumination direction and directedness from the appearance of objects, first for simple rendered Lambertian smooth white spheres and, second, for real objects with arbitrary reflectances and surface structures. In this article, we will study perception of the spatial radiometric properties of the light field, not of the spectral properties of the illumination.

The light field (Gershun, 1939; Moon \& Spencer, 1981) is a complete global description of the radiance as a function of position and direction and is thus different from the illuminance, which is a local description at the surfaces of objects. The illumination direction and directedness or degree of collimation are low-order light field properties (in a mathematical sense, e.g., the "ambient" illumination and the illumination direction correspond with the zeroth and first order terms in a spherical harmonics development). Manipulating just these lower order properties, one can create important canonical cases of light fields (Adams, 1981; Gershun, 1939; Hunter \& Fuqua, 1990; Kahrs, Calahan, Carson, \& Poster, 1996). These canonical cases include direct sunlight, which has a high degree of collimation and a direction that is often quite clear because of harsh shadowing effects. An overcast sky is not that directed, but the illumination direction can still be derived from the fact that roughly ovoid objects have light poles and dark poles. Completely diffuse illumination happens rarely, but in a polar white-out, for instance, the illumination would not be directed at all. Interestingly, these global properties of light fields do not vary much from one position to another in many natural scenes, especially outside scenes (contradictory to high frequency variations of natural illumination). Therefore, if one would be able to estimate the light field (e.g., from the appearance of an object in that scene), it would provide one with a radiometric framework or an es-

S. C.Pont, s.c.pont@phys.uu.nl 


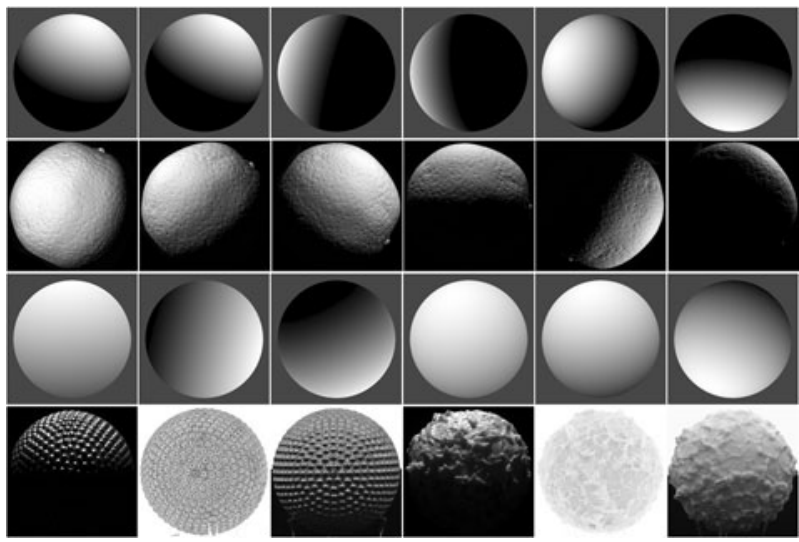

Figure 1. Examples of stimuli. The stimuli that were used in Experiments 1 and 2 are shown in the first and second rows and the third row, respectively. First row: Lambertian spheres, illuminated with a collimated beam from arbitrary directions distributed homogeneously in the ranges azimuth 0 to 360 degrees and polar 0 to 120 degrees. Second row: For just one sample (Sample 3, an orange painted matte white), we show photographs that were made under collimated illumination and rotated over random angles. For each of the 22 objects, we used 16 images: four random rotations or azimuths times four polar angles $(30,60,90$, and 120 degrees; of course, the polar angles could not be varied using image processing techniques). Third row: Lambertian spheres, illuminated with a beam from arbitrary directions distributed homogeneously in the ranges azimuth 0 to 360 degrees and polar angle 0 to 120 degrees, with the directedness or degree of collimation varying from fully diffuse (or "Ganzfeld illumination," similar to conditions such as a polar white-out; directedness value -1 ), via hemispherical diffuse (similar to illumination by an overcast sky; directedness value 0 ), to fully collimated (similar to direct sunlight; directedness value 1). Fourth row: For just two samples (Samples 30 and 32, a blobby Christmas ball and a candle with a very rough finish), we show the photographs for the different levels of directedness that were available - collimated 90 degrees, hemispherical diffuse 90 degrees, and Ganzfeld illumination.

timate of the "luminous atmosphere" (Adelson \& Bergen, 1991; Marr, 1982). The perception of these lower order properties of the light field might serve as a baseline for the derivation of shape, reflectance, and surface structure of objects. Some researchers have shown that observers are able to judge the (collimated) illumination direction from isolated globally flat patches of textures (Koenderink, van Doorn, Kappers, te Pas, \& Pont, 2003; Koenderink, van Doorn, \& Pont, 2004) and that a prior for light-from-above exists (Mamassian, Landy, \& Maloney, 2002). In this article, we examine the perception of spatial lower order properties of the light field from 3-D objects.

\section{EXPERIMENT 1}

In the first part of Experiment 1, we tested whether observers were able to match the illumination over two rendered smooth Lambertian (Lambert, 1760) spheres of unit albedo that were illuminated by a collimated white beam on a blue background. In the second part of Experiment 1 , we tested whether participants were able to match the illumination direction of a smooth Lambertian sphere (the probe) with that of a real, approximately spherical, rough, non-Lambertian object. We expected that performance for matching spheres of different materials would be worse than that for spheres of the same materials, because the inverse problem (inferring the light field and/or material properties and/or shape from 2-D images) is basically underdetermined. Furthermore, we were interested in whether systematic deviations would occur for specific materials. Part 1 of Experiment 1 served as a baseline experiment in several respects. First, we wanted to know whether human observers can match the normal component of the surface illumination. The radiance distribution in the images of smooth Lambertian spheres corresponds directly to the normal component of the surface illumination, because the bidirectional reflectance distribution function (BRDF; Nicodemus, Richmond, Hsia, Ginsberg, \& Limperis, 1977) is a constant. Second, we need the data for comparison with cases in which illumination of Lambertian spheres was compared with illumination of real objects of arbitrary materials ("probe characterization"). Note that for real objects, the BRDF is non-Lambertian (CUReT database, 1999; Dana, van Ginneken, Nayar, \& Koenderink, 1999) and, generally, natural object surfaces are corrugated, causing bidirectional texture gradients (Pont \& Koenderink, 2005) that reveal the component of the illuminance, that is parallel to the global object surface, or the surface illuminance flow (Pont \& Koenderink, 2004).

\section{Method}

Stimuli. The test images of Lambertian spheres (see Figure 1, first row) were rendered for randomized illumination directions of a fully collimated beam. A fully collimated beam means that all light rays come from exactly the same direction, which is sometimesmistakenly - called point source at infinity. The azimuths were drawn from a uniform distribution on $\left(0^{\circ}, 360^{\circ}\right)$. The polar angles were drawn from a probability density function $\sin 2 p$ (with $p$ the polar angle) on $\left(0^{\circ}, 120^{\circ}\right)$. This was done in order to prevent the occurrence of an overdose of grazing samples. In the neighborhood of the zenith (normal incidence), the probability density is nearly uniform on the hemisphere of illumination directions. We rendered 400 images, which were all presented once to each observer.

The test images of real objects were photographs, which were chosen from the Utrecht oranges database (Pont \& Koenderink, 2004). This database consists of photometrically calibrated images of approximately spherical objects with different reflectances, textures, and even translucencies. The database contains six photographs per object (see Figure 2, last row): four under collimated illumination (similar to direct sunlight) from angles differing $30^{\circ}$, $60^{\circ}, 90^{\circ}$, and $120^{\circ}$ from the viewing direction, one under hemispherical diffuse illumination (similar to illumination by an overcast sky), and one under Ganzfeld or fully diffuse illumination (similar to a polar white-out condition). The objects that were selected from the database are shown in Figure 2 in the upper four rows for collimated illumination under $120^{\circ}$ only. In Experiment 1, we used the images that were made under collimated illumination; thus, per object, the illumination could be from four polar angles $\left(30^{\circ}, 60^{\circ}, 90^{\circ}\right.$, and $120^{\circ}$; for examples, see Figure 2, lower row, the first four images from the left). In order to vary the azimuthal angle, we rotated each of those images four times over random angles. Thus, we presented 16 images per object (for examples, see Figure 1, second row), which adds up to 16 images $\times 22$ objects $=352$ stimuli (each observer performed one judgment per stimulus). All test images were $512 \times$ 512 pixels, grayscale TIFF-formatted files.

Observers. Four observers participated in the experiments: the first author and 3 observers who were naive with regard to our re- 


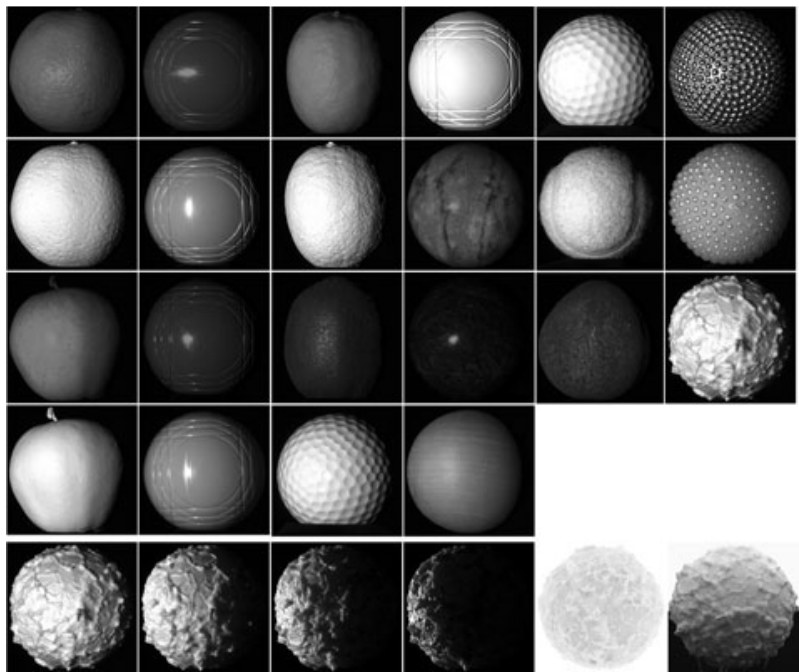

Figure 2. A representation of the 22 objects (upper rows) and 6 illumination conditions (last row) in the selection of 132 images ( 22 objects $\times 6$ photographs) from the Utrecht oranges database (Pont \& Koenderink, 2004), which was used in this article. The files in the database were calibrated photometrically, and for the experiments in this article, we used a monitor with linear gamma; the printed versions probably look different from the stimulus presentations on screen.

search. The naive observers were paid for their efforts. All observers had normal or corrected-to-normal eyesight

Setup. The setup consisted of an Apple Macintosh Dual 2-GHz PowerPC G5 and a luminance linearized 22-in. LaCie Blue Electron monitor at $75 \mathrm{~Hz}$ and $1,600 \times 1,200$ pixels. Participants were seated with their heads in a chinrest that was $83 \mathrm{~cm}$ from the screen and viewed the images monocularly. The stimulus and probe images extended for visual angles of $8.5^{\circ} \times 8.5^{\circ}$ each.

Design and Procedure. Participants were asked to match the illumination direction using an interactive interface in which the stimulus and matched image were shown in addition to a projection of the matched direction (see Figure 3). In Experiment 1, the slider at the left was not visible for the observers. The upper left sphere in Figure 3 was the stimulus; a Lambertian sphere was in the first part, and a photograph of a real object was in the second part of Experiment 1. Observers could adjust the illumination direction for the rightmost sphere (the probe) interactively by means of dragging the computer mouse. The design was such that the fast, live probe rendering covaried simultaneously during the setting by the observer. The rendering of the Lambertian sphere of course simply followed Lambert's law; thus, the local radiance was computed according to $\vec{i} \cdot \vec{n}$, with $\vec{i}$ being the normalized vector representing the illumination direction and $\vec{n}$ being the local surface normal. Delays between dragging the mouse and the rendering of the sphere were not noticeable (using the G5 computer; our G4 did not suffice). In the projection at the bottom right, a red dot representing the beam direction was shown for reference. Observers could take as much time as they needed to match the illumination of the two spheres. To explain the task to naive observers, we used a spotlight and a real matte white sphere (Part 1) and a spotlight and real objects - namely, a matte white sphere, a specular smooth sphere, a glossy smooth sphere, and a rough matte sphere (Part 2). Participants were asked to match the illumination directions and were explicitly told not to match the image structures.

\section{Results and Discussion}

In Figure 4, the results for Experiment 1 are shown for all 4 observers. The results for Experiment 1 for the Lam- bertian spheres are depicted by means of black dots, and for the real objects by gray dots. We show the settings against the stimulus values (thus, perfect settings would result in dots on a straight line with unit slope through the origin [offset zero]) for the azimuthal angles in the upper half and for the polar angles in the lower half of Figure 4. Additionally, in Table 1, the results are shown of linear regressions (offsets, slopes, and $R^{2} \mathrm{~s}$ ) of the data for all observers, for the Lambertian and the real objects, and for the polar and the azimuthal angles, separately. The results reveal that observers were able to match the illumination directions of Lambertian spheres very well. From the data for the Lambertian spheres (the black dots), it is clear that the response and stimulus values correlate very well and that variance is very low. The regression results indeed show that offsets are negligible, slopes are approximately one, and $R^{2} \mathrm{~s}$ are very high. From the data for the real objects (the gray dots), it is clear that the azimuthal settings for real objects result in correlations and variances similar to those of the settings for Lambertian artificial spheres. The polar settings, however, do show larger variances for real objects than for Lambertian spheres. The regression results confirm these pictures. Thus, reflectance, texture, transparency, and albedo differences do not influence the perceived illumination orientation, in contradistinction to the perceived polar angle of the illumination.

Furthermore, we found specific outliers for real objects, and the result is depicted in Figure 5. This figure shows

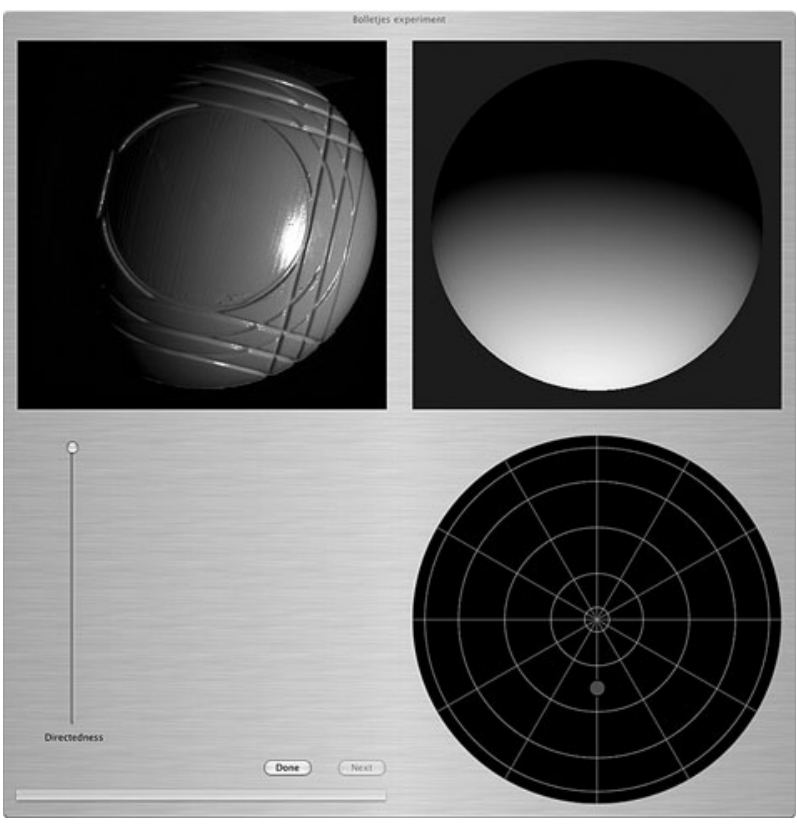

Figure 3. The interactive interface: Above left, a stimulus; above right, the response view; and below right, the projection of the illumination direction. Below left a slider is visible, which was used in Experiment 2 for adjustment of the directedness of the beam. Furthermore, a progression bar was shown (horizontal bar below left), and there are two buttons that observers had to activate after matching a stimulus: a "done" button and a "next" button. 

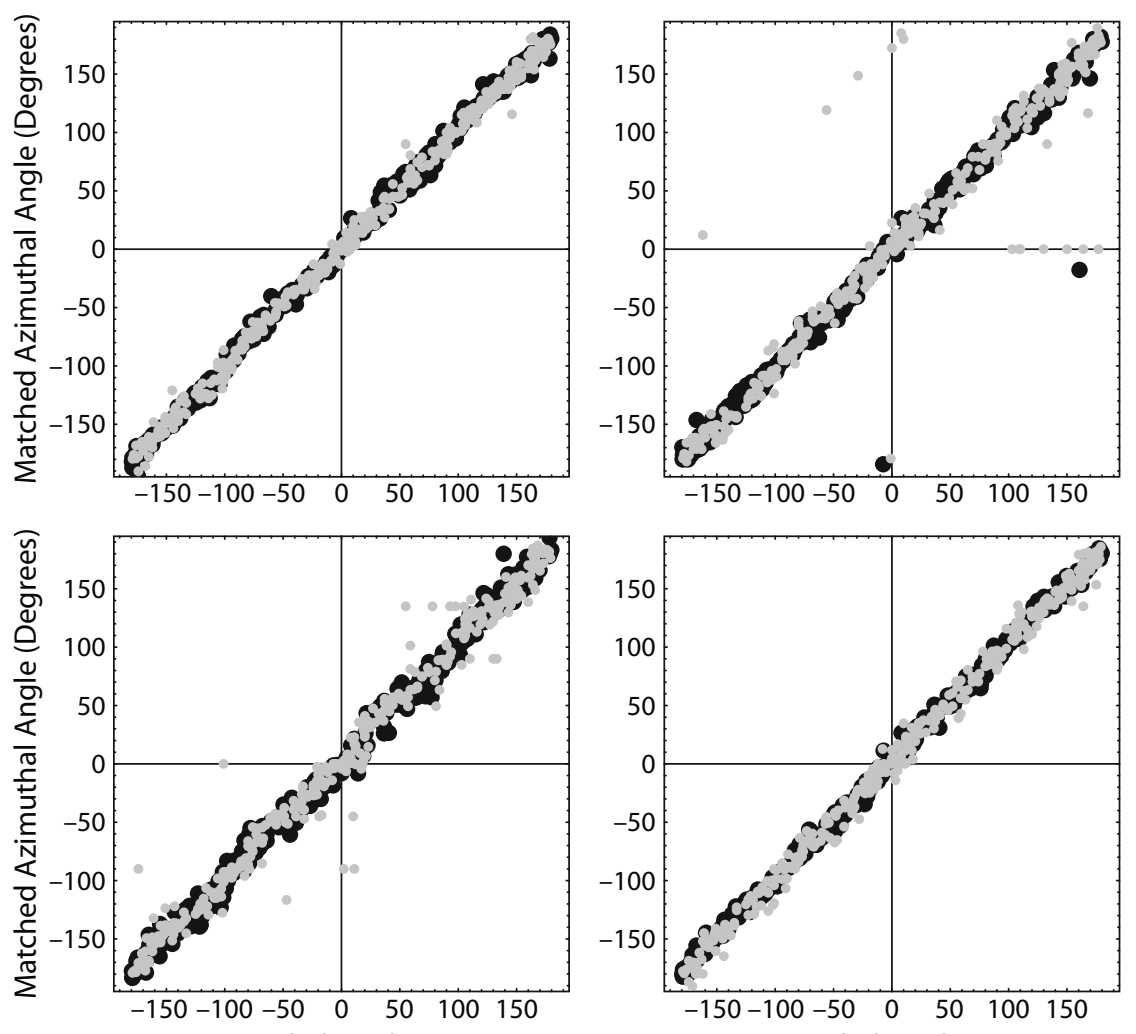

Test Azimuthal Angle (Degrees)
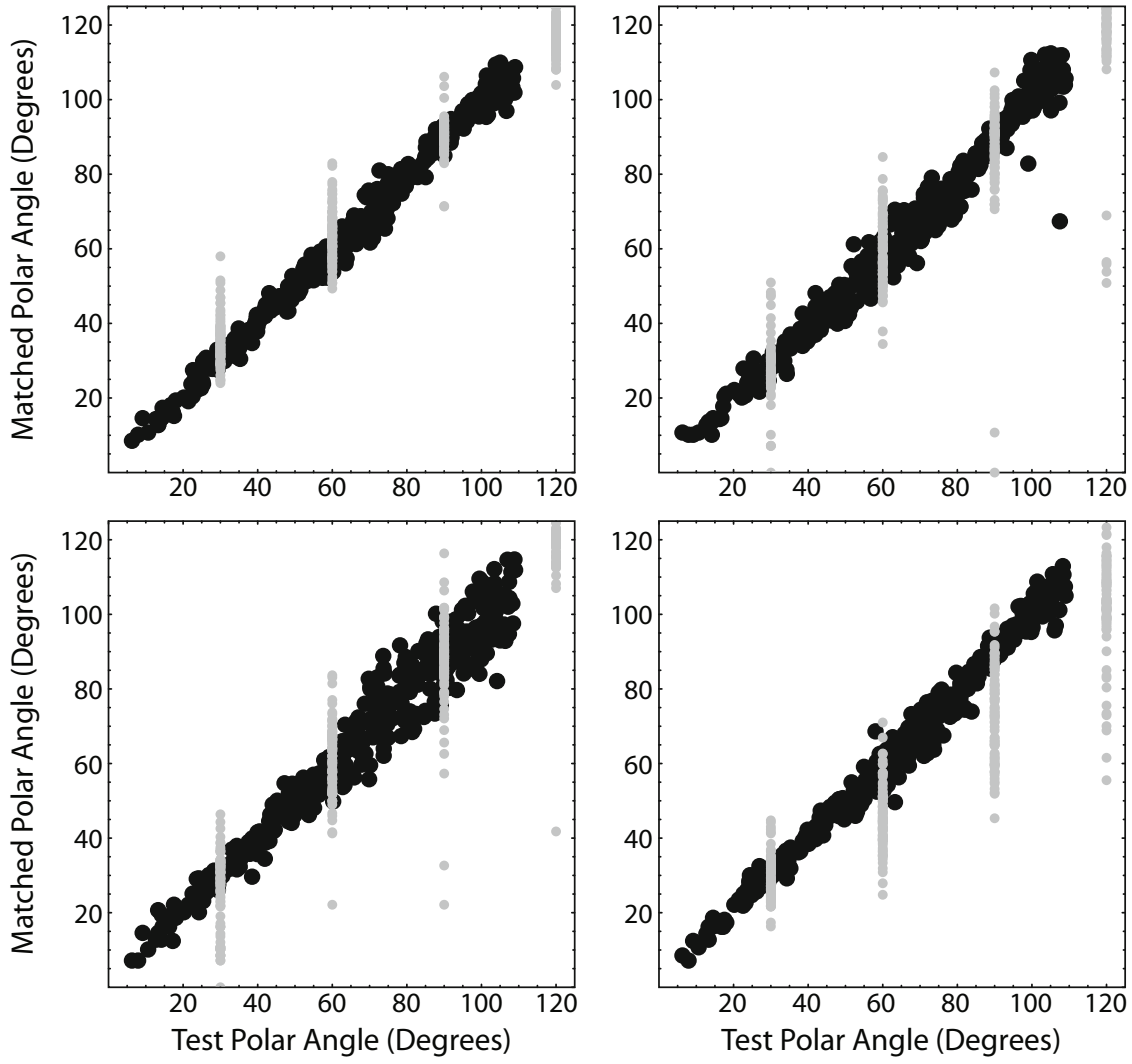

Figure 4. The results of Experiment 1 for Lambertian rendered spheres (black dots) and for real objects (gray dots). The settings for the azimuthal and polar angles of the illumination directions are depicted in the upper and lower four graphs, respectively, as a function of the stimulus values. Results for Observers S.P., N.K., C.L., and D.R. are depicted in the upper left, upper right, lower left, and lower right graphs, respectively. 
Table 1

Regression Results of the Data of Experiment 1 for All 4 Observers

\begin{tabular}{crrrrrrrr}
\hline Observer & \multicolumn{1}{c}{ Lamb., Polar } & $R^{2}$ & Lamb., Azimuth & \multicolumn{1}{c}{$R^{2}$} & \multicolumn{1}{c}{ Real, Polar } & \multicolumn{1}{c}{$R^{2}$} & Real, Azimuth & $R^{2}$ \\
\hline S.P. & $0.57+0.98 x$ & .99 & $1.37+1.01 x$ & 1.00 & $8.03+0.92 x$ & .96 & $1.00+1.01 x$ & 1.00 \\
N.K. & $-2.06+1.01 x$ & .97 & $-0.83+1.00 x$ & .97 & $-1.99+0.99 x$ & .87 & $-0.35+0.97 x$ & .90 \\
C.L. & $1.37+0.96 x$ & .96 & $2.62+1.01 x$ & .99 & $-4.21+1.04 x$ & .91 & $2.80+1.02 x$ & .96 \\
D.R. & $0.34+0.99 x$ & .99 & $2.58+1.00 x$ & 1.00 & $0.71+0.83 x$ & .86 & $1.54+1.02 x$ & .99 \\
\hline
\end{tabular}

Note-Each pair of columns shows the fitted line (offset + slope $* x$, with $x$ being the test angle in degrees) and the $R^{2}$ value. From left to right, the results are shown for the Lambertian (Lamb.) spheres for the polar and azimuthal angles and for the real images for the polar and azimuthal angles.

the number of times that objects were found to be outliers in the data, over all stimulus presentations and observers (64 per object). Outliers were defined as those datapoints for which the angle between the stimulus direction and the matched direction was larger than twice the standard deviation of that angle. The stars depict those objects that were found to be outliers for all observers. Consistently, we found translucent objects (Numbers 22, translucent yellow plastic jeu de boulles game ball; 31 , translucent white candle with bumps and pearly finish; and 32, translucent white candle with very rough finish; the numbers refer to the object numbers in the Utrecht oranges database) and the fluorescent hairy tennis ball (Number 14) to be outliers in the data.

\section{EXPERIMENT 2}

Except for the case of direct sunlight, in natural scenes the illumination is hardly ever fully collimated (Dror et al., 2004; Gershun, 1939). Even more exceptional is the case of fully diffuse illumination - for example, a polar white-out. Most often, the directedness of the illumination is somewhere between these two extremes. Therefore, in Experiment 2, we tested whether observers were able to simultaneously match the direction and directedness of the illumination of two rendered Lambertian spheres. We expected that the addition of the extra degree of freedom to the design would increase variability of the settings and possibly perceptual interactions because of basic ambiguities in the solution of the inverse problem.

\section{Method}

Stimuli. We rendered Lambertian spheres. The directions were chosen randomly in the same way as those in Experiment 1. The directedness was randomly varied between -1 and 1 - that is, between fully diffuse and fully collimated. A directedness of 0 represents hemispherical diffuse illumination. The rendering of the Lambertian sphere was done via the ambient plus collimated beam approach, or, in other words, a superposition of zeroth and firstorder terms of a spherical harmonics development of the light field (monopole plus dipole). Thus, the local radiance was computed according to $[(1-$ directedness $)+(1+$ directedness $)(\vec{i} \cdot \vec{n})] / 2$, with $\vec{i}$ being the normalized vector representing the illumination direction and $\vec{n}$ being the local surface normal. In the third row of Figure 1, we show some examples of stimuli. Altogether, we rendered 400 stimuli (all observers performed one judgment per stimulus).

Observers. The same 4 observers who participated in Experiment 1 also participated in Experiment 2.

Setup. In Experiment 2, we used the same setup as that in Experiment 1.

Design and Procedure. Participants were asked to match the illumination direction and directedness using the interactive interface again; see Figure 3. In Experiment 2, the slider at the left was visible for the observers. This slider could be adjusted interactively such that

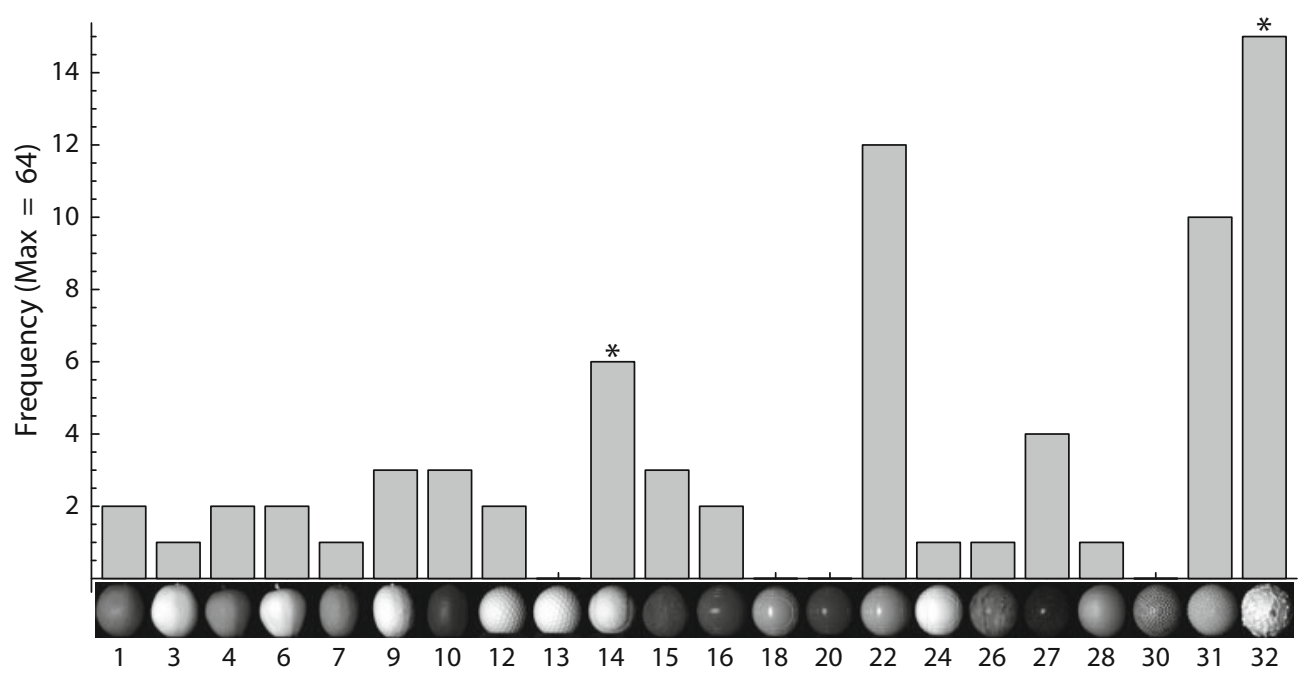

Figure 5. The outliers that were found in the results for Experiment 1. The graph depicts the number of times that an object was found to be an outlier, for all observers and all presentations (maximum 64), as a function of the object number in the Utrecht oranges database. For the reader's convenience, we also depict an icon representing the object. The stars signal objects that were found to be outliers for all observers. 

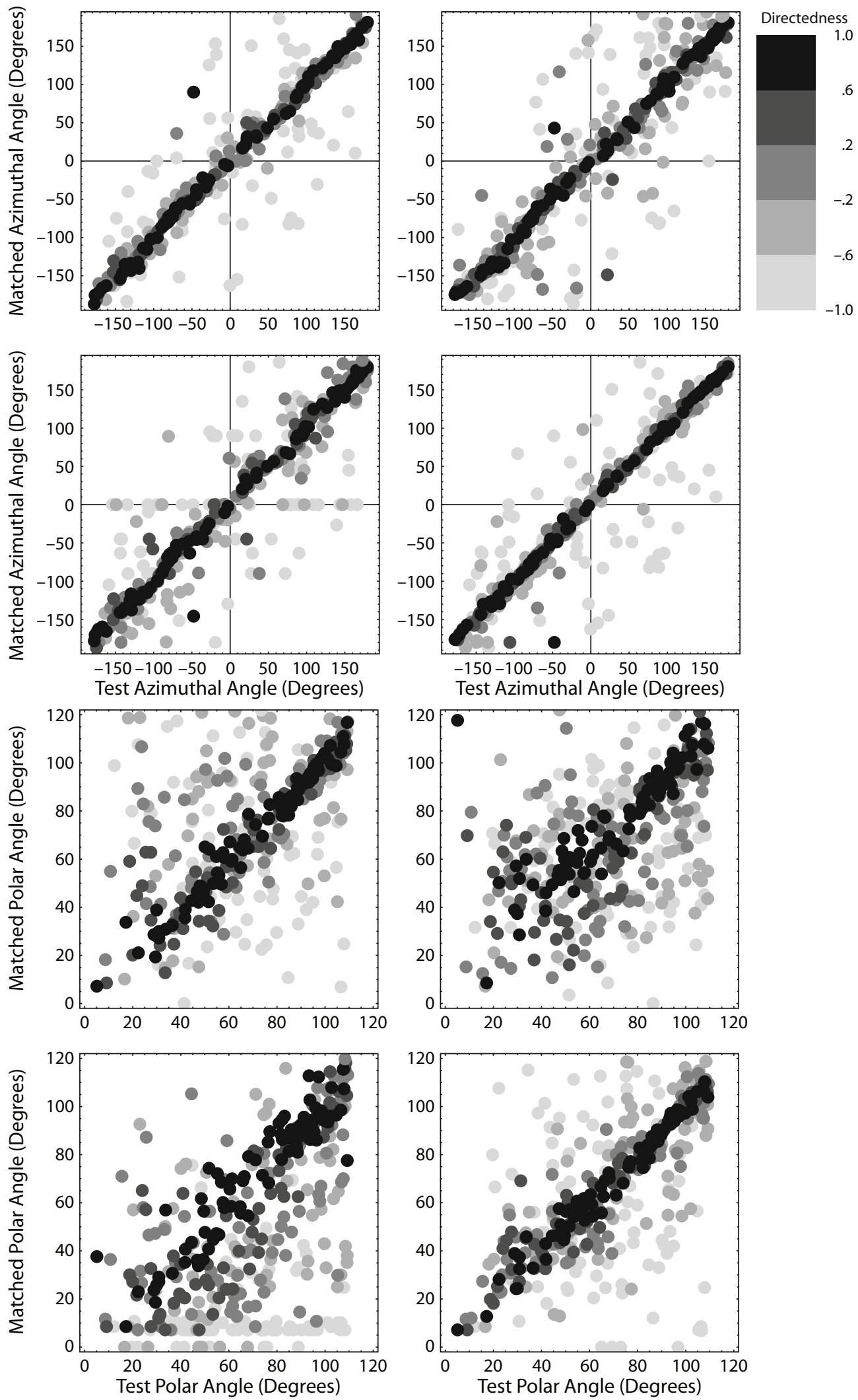

Figure 6. The results for the angle settings in Experiment 2 as a function of stimulus values. The upper and lower four graphs depict results for the azimuthal and polar angle settings, respectively. The dots are gray-level coded according to the directedness value of the stimulus following the scale above right; for example, black dots represent data in the "very directed beam" regime and light gray dots represent data in the "very diffuse beam" regime. Results for Observers S.P., N.K., C.L., and D.R. are depicted in the upper left, upper right, lower left, and lower right graphs, respectively. 
the lowest level represented fully diffuse illumination (directedness -1 ), the middle level represented hemispherical diffuse illumination (directedness 0 ), and the highest level represented fully collimated illumination (directedness 1). Except for the extra setting of the directedness slider, the task was the same as that in Experiment 1.

\section{Results and Discussion}

In Figures 6-8, we depict the results of Experiment 2. The data for the direction settings in Figure 6 were split in five regimes for stimulus directednesses between -1 and -.6 (light gray points, very diffuse illumination), -.6 and -.2 (quite diffuse), -.2 and .2 (middle gray points, approximately hemispherical diffuse), .2 and .6 (quite directed), and .6 and 1 (black points, very collimated beams). We show the settings as a function of the stimulus values for the azimuthal angles (upper four graphs) and the polar angles (lower four graphs). It is clear that for very directed beams (black dots), the illumination direction was matched quite closely; these results are similar to the data of Experiment 1. However, if the illumination becomes more diffuse (and the dots' colors change to a lighter gray), we find that the direction of the source was matched less well. In other words, the variance in the directional settings decreases as a function of the directedness. We calculated the standard deviation for the polar and azimuthal angles, for the five directedness regimes and for all observers separately. In all cases, we found a steep decrease of the standard deviation as a function of the directedness; polar deviations decreased from about 40 to about 8 and azimuthal deviations decreased from about 95 to about 14 .
The settings for the directedness itself, which are shown in Figure 7 as a function of the stimulus values, do not show such a trend. The directedness settings correlate well with the stimulus values although they overall show quite a large variance. We did regression analyses for the polar, azimuthal, and directedness settings, which are shown in Table 2, and also for those data for the five directedness regimes separately. The regression results confirmed the figures described above and, furthermore, they clearly show that in comparison with the results of Experiment 1, overall variability of the data increased. This increased variability is mainly due to the settings for quite diffuse and very diffuse illumination; regression results for the very collimated regime were similar to the results of Experiment 1 . We checked for other correlations in the data and found one more significant relation, which is shown in Figure 8. Here, the errors in the directedness are shown as a function of the errors in the polar angles of the direction. These data show slight but significant (on a $p<.05$ level) negative correlations for all observers.

\section{GENERAL DISCUSSION}

In Experiment 1, we found that observers are very well able to match the illumination direction of two rendered Lambertian spheres under collimated illumination and that observers are also quite able to match the illumination azimuth of a rendered Lambertian sphere with that of a real approximately convex object of arbitrary reflectance and surface structure (texture). The azimuth of the
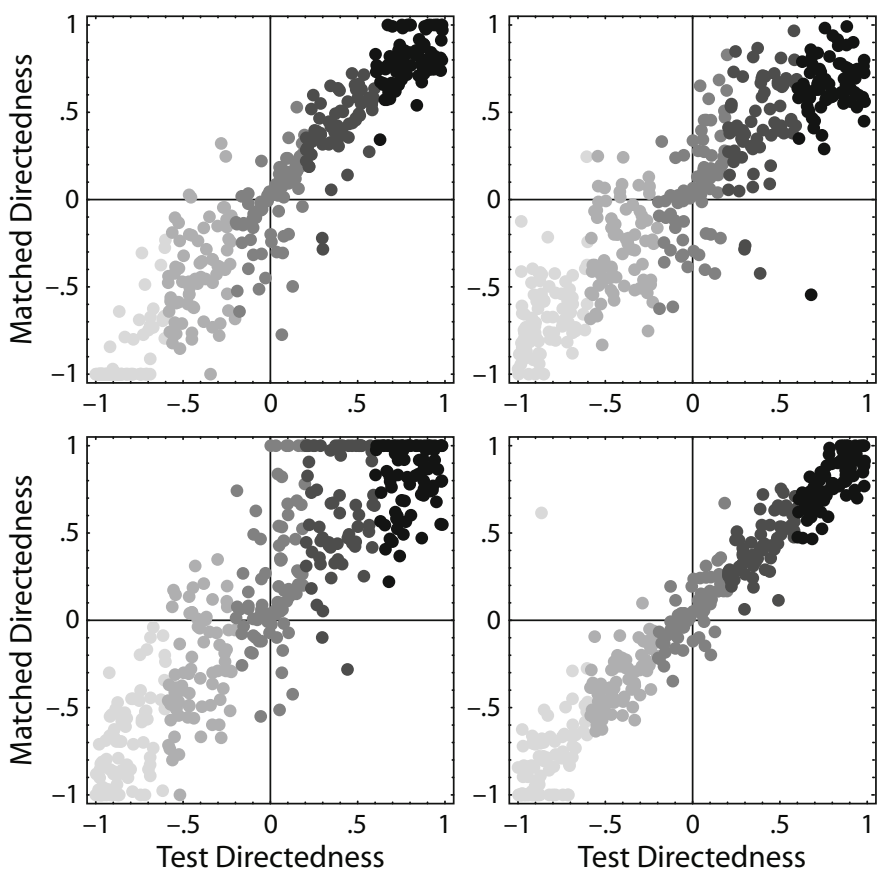

Figure 7. The results for the directedness settings in Experiment 2, as a function of stimulus values. The dots are gray-level coded in the same manner as those in Figure 6. Results for Observers S.P., N.K., C.L., and D.R. are depicted in the upper left, upper right, lower left, and lower right graphs, respectively. 
Table 2

Regression Results of the Data of Experiment 2 for All 4 Observers

\begin{tabular}{crrrrrr}
\hline Observers & Polar & $R^{2}$ & \multicolumn{1}{c}{ Azimuthal } & $R^{2}$ & \multicolumn{1}{c}{ Directedness } & $R^{2}$ \\
\hline S.P. & $41.27+0.55 x$ & .18 & $0.74+1.02 x$ & .85 & $-0.02+1.04 x$ & .93 \\
N.K. & $32.98+0.51 x$ & .25 & $-0.72+1.06 x$ & .82 & $0.05+0.82 x$ & .82 \\
C.L. & $2.02+0.68 x$ & .27 & $3.29+0.99 x$ & .79 & $0.14+0.97 x$ & .81 \\
D.R. & $14.94+0.78 x$ & .43 & $-2.45+1.01 x$ & .84 & $0.03+0.97 x$ & .95 \\
\hline
\end{tabular}

Note-Each pair of columns shows the fitted line (offset + slope $* x$, with $x$ being the test angle in degrees or the directedness) and the $R^{2}$ value. From left to right, the results are shown for the polar and azimuthal angles and for the directedness.

illumination direction might be guesstimated by means of symmetries in the isophote patterns, which are quite independent of material reflectance and texture gradients (Note: We only used objects with isotropic surface structures). The polar settings showed a larger variance for real objects. The polar angle of the beam direction might be guesstimated on the basis of a combination of several cues, some of which are strongly influenced by material properties. For example, the position of the brightest area on the surface of the object might be used as a cue. Glossy surfaces show a highlight structure at the so-called specular position; thus, for glossy objects, the brightest areas are at different locations than the brightest areas on matte objects (which are at the positions where the beams hit the surfaces head on). So, if the brightest area were used as a cue for the illumination direction, the polar angle would be matched too low for glossy surfaces.

Furthermore, the position of the shadow boundary might be used as a cue (Baxandall, 1995; Hogarth, 1981; Jacobs, 1988; Mamassian, Knill, \& Kersten, 1998). Trans- lucent objects were found to be outliers in Experiment 2. This result might be due to the fact that for these objects, the light diffuses through the object into the shadow area, so there is no sharp shadow boundary visible. The fluorescent hairy tennis ball was also found to be an outlier in Experiment 1, which might also be caused by photometrical effects on the shadow boundary, although the cause must be different. In the case of the tennis ball, the hairy interface causes asperity scattering (Koenderink \& Pont, 2003) into the shadow region.

Finally, the fact that in Experiment 1 the variance in the data was larger for real objects than for Lambertian spheres may be due to the difference in visibility of the back ground; for the rendered spheres of Experiment 1, the background was blue and clearly visible, whereas for the real objects in Experiment 1, the background was hardly visible. Therefore, the relative position of the shadow boundary might have been more difficult to infer, which might have affected the settings. On the other hand, the variances in the settings for the azimuth were similar and
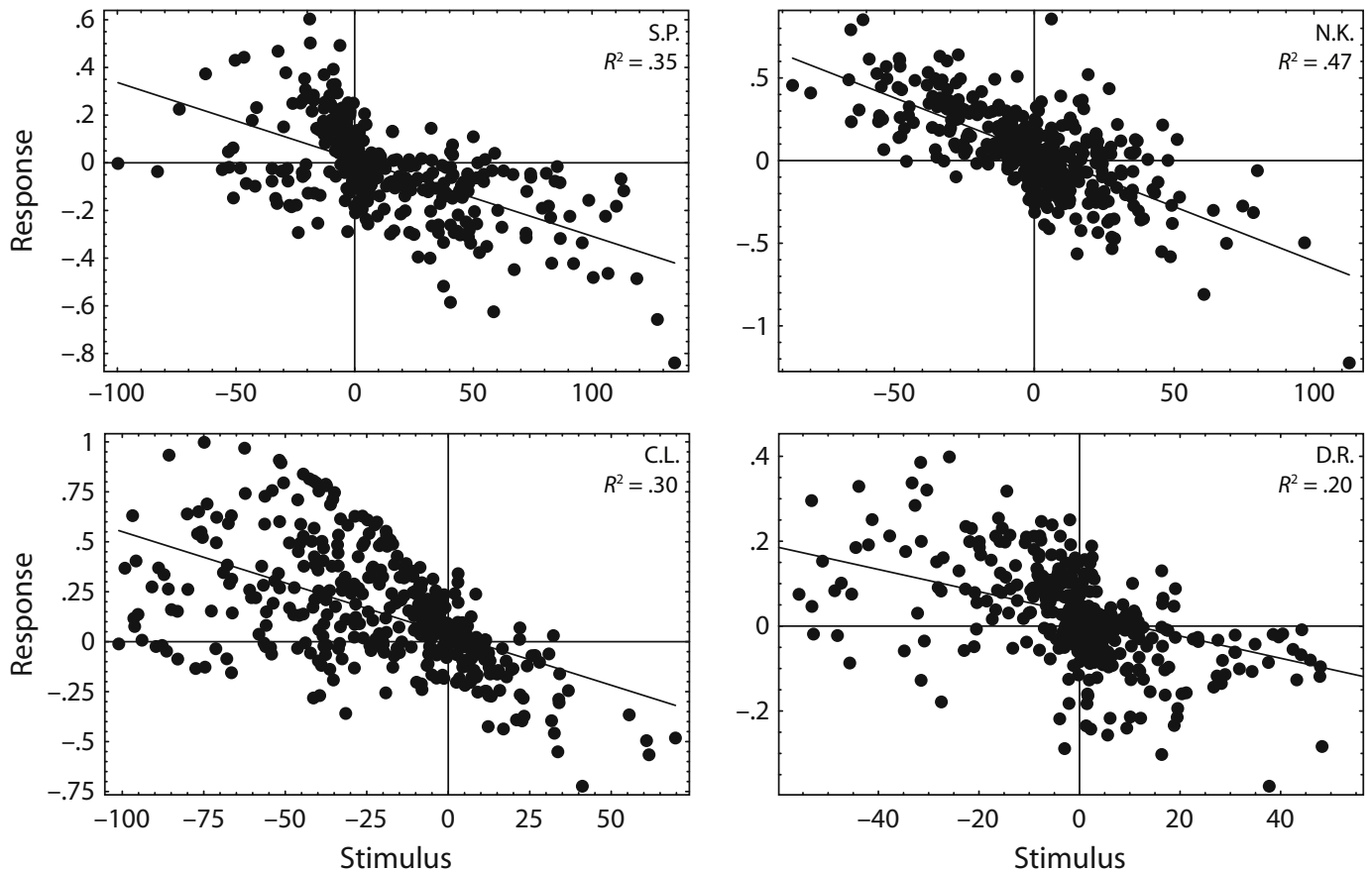

Figure 8. The errors in the directedness settings in Experiment 2 as a function of the errors in the polar angle settings. 


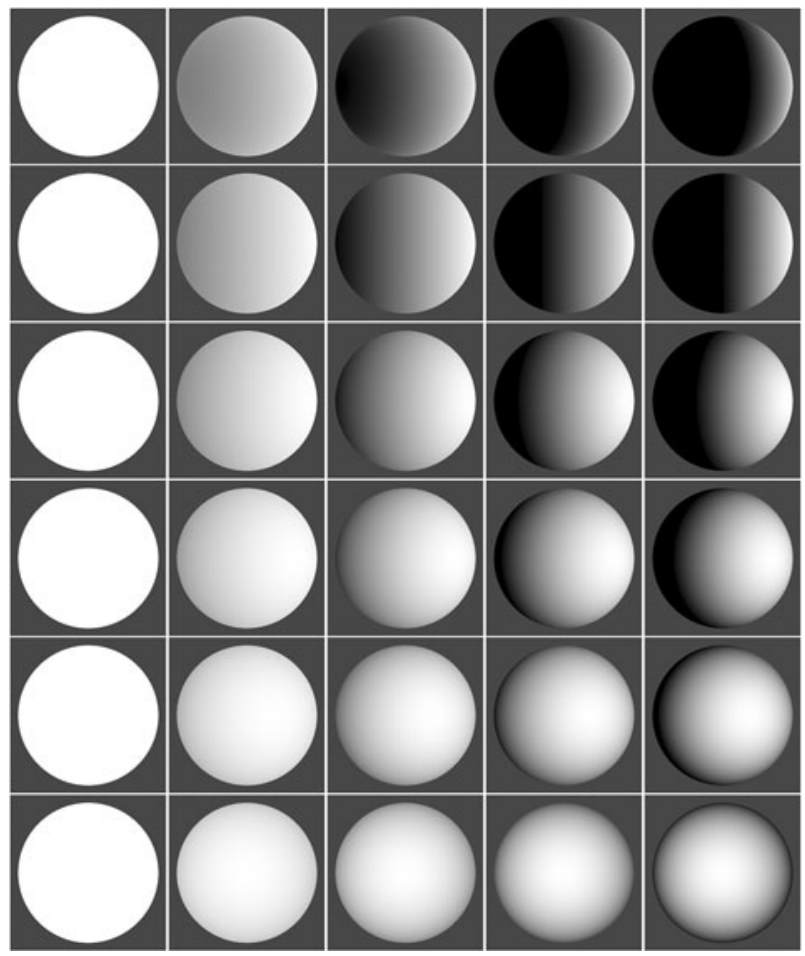

Figure 9. Rendered Lambertian spheres for which the directedness and polar angle were varied systematically (azimuth was always 0): From left to right, the directedness varies from -1 to 1 in steps of .5 , and from the top to the bottom, the polar angle varies from $112.5^{\circ}$ to $0^{\circ}$ in steps of $22.5^{\circ}$. Notice that variations along the diagonals from above left to below right result in illuminance patterns that are more similar than those along other directions.

quite small for real and for rendered spheres, so if there was an influence of background, this was primarily on the polar settings.

When the illumination direction and directedness (or "degree of collimation," or "width of the beam") had to be matched for Lambertian rendered spheres, the variance in the illumination direction settings was larger if the illumination was more diffuse. This effect is partly due to an interaction between the directedness and the polar angle settings: There was a trend in the data that if the directedness was set too low, the polar angle was set too high. In Figure 9, we show a systematically parameterized set of Lambertian spheres that explains this effect. Bottom to top, the polar angle increases, and left to right, the directedness increases. Although the steps are quite large in this figure, it is clear that images along the top left to bottom right diagonals are much more similar than images along other directions. Thus, the perceptual interaction corresponds with correlations in object appearance for inversely related polar angles and directedness. If this conclusion can be extrapolated to light field perception in natural scenes, the consequence is that human observers' radiometric frameworks might be far from veridical, especially for very diffuse illumination. Furthermore, if such a framework served as a baseline for the derivation of object properties, then shape, reflectance, and/or surface structure perception would also deviate from veridical in a systematic way.

Unfortunately, our "oranges database" was too limited for a thorough comparison of the results for rendered artificial spheres and for photographs of real objects (it contains only three images per object for directedness variations; see Figure 1, last row, and Figure 2). In order to proceed with these experiments, we need a more extended database, preferably one that contains a dense sampling of polar angles and degrees of collimation.

These experiments both started with the question of whether we can derive light field properties from the appearance of objects in scenes. We showed that observers are able to match the illumination of Lambertian spheres with Lambertian spheres and with real objects and that there are effects of material (especially translucency) and of the directedness of the beam. Of course, probing the illumination by means of matching the illumination of a Lambertian sphere is rather indirect, and one might question whether observers matched the light that was reflected by the object or the light that illuminated the object. We took care to instruct the observers to match the illumination and not the image structure. If observers had matched the image structure instead of the illumination, we would have found hardly any correlation plus different outliers for the real objects in Experiment 1; for example, the four very dark and glossy or even shiny objects and the silvery blobby Christmas ball show isophote patterns that differ very much from Lambertian spheres. So, observers could not have simply matched the test and stimulus on the basis of straight correlations between test and stimulus images, but must really have tried to match the perceived illumination direction. Nonetheless, in future research, we will conduct experiments in which observers will interactively adjust the illumination in a real scene.

On the other hand, we expect that image structure and judgments of illumination are confounded, because the inverse problem is basically underdetermined. Generally projected illuminance patterns (on an image plane and on the retina) are probably the primary (but indirect) cue for judgments of the illumination, whereas we often avoid looking straight at light sources. Furthermore, if projected illuminance patterns are the primary cue for the light field, we would need to be able to perfectly estimate and reckon with material properties in order to derive the light field from the projected illuminance pattern. However, in order to estimate the material properties from the illuminance pattern, we of course need an estimate of the light field (Note: We do not share the general opinion that we can take "material constancy" for granted [te Pas \& Pont, 2005; te Pas, Pont, \& Paffen, 2004]). Another complication is that an estimate of the shape would also be needed to derive these properties from the image structure. Human perception of local shape from shading under variable lighting (collimated and diffuse) was shown to suffer from inherent ambiguities between shape and shading that depend on lighting (Langer \& Bülthoff, 2001). Important topics in this regard concern "priors" and statistics of natural scenes (Dror et al., 2004; Fleming, Dror, \& Adelson, 2003; Langer \& Bülthoff, 2001; Mamassian 
et al., 1998), which might serve as constraints to the inverse problem.

In conclusion, if one takes into account that in the derivation of physical properties from images there exist many basic ambiguities between estimates of those physical properties, it actually is surprising that we seem to be able to perform so well on such tasks. Because the field of "ecological optics" (Gibson, 1950) is still in its infancy, we might expect that in the future we will get a better grip on the basic mechanisms that underlie these processes. For instance, local interreflections, global scene interaction, illuminance flow, and the statistics of natural scenes and light fields might at least provide us with extra constraints on the solutions.

\section{AUTHOR NOTE}

This work was supported by the Netherlands Organization for Scientific Research and the 3-D Shape and Material Properties for Recognition project, as well as the InSight $2^{+}$project (Information Society Technologies Programme IST-2000-29688). Address correspondence to S. C. Pont, Helmholtz Institute, Department of Physics and Astronomy, Utrecht University, Princetonplein 5, 3584 CC Utrecht, The Netherlands (e-mail: s.c.pont@phys.uu.nl).

\section{REFERENCES}

Adams, A. (1981). The negative: The Ansel Adams photography series 2. Boston: Little, Brown.

Adelson, E. H., \& Bergen, J. R. (1991). The plenoptic function and the elements of early vision. In M. Landy \& J. A. Movshon (Eds.), Computational models of visual processing (pp. 3-20). Cambridge, MA: MIT Press.

Adelson, E. H., \& Pentland, A. P. (1996). The perception of shading and reflectance. In D. Knill \& W. Richards (Eds.), Perception as Bayesian inference (pp. 409-423). Cambridge: Cambridge University Press.

BaXANDAll, M. (1995). Shadows and enlightenment. New Haven: Yale University Press.

Belhumeur, P. N., Kriegman, D., \& Yuille, A. (1999). The bas-relief ambiguity. International Journal of Computer Vision, 35, 33-44.

CUReT database (1999). Columbia-Utrecht reflectance and texture database. Retrieved March 1, 2003, from www.cs.columbia .edu/CAVE/curet.

Dana, K. J., van Ginneken, B., Nayar, S. K., \& Koenderink, J. J. (1999). Reflectance and texture of real-world surfaces. ACM Transactions on Graphics, 18, 1-34.

Dror, R. O., Willsky, A. S., \& Adelson, E. H. (2004). Statistical characterization of real-world illumination. Journal of Vision, 4, 821-837.

Fleming, R. W., Dror, R. O., \& Adelson, E. H. (2003). Real-world illumination and the perception of surface reflectance properties. Journal of Vision, 3, 347-368.

Gershun, A. (1939). The light field [P. Moon \& G. Timoshenko, Trans.]. Journal of Mathematics \& Physics, 18, 51-151.

Gibson, J. J. (1950). The perception of the visual world. Boston: Houghton Mifflin.
Hogarth, B. (1981). Dynamic light and shape. New York: Watson-Guptill. Hunter, F., \& FUQUA, P. (1990). Light: Science and magic. An introduction to photographic lighting. Boston: Focal Press.

InSight2 ${ }^{+}$Project. Information Society Technologies Programme IST-2000-29688. Retrieved March 1, 2003, from www-sop.inria.fr/ robotvis/projects/Insight2+/presentation.html.

JACOBS, T. S. (1988). Light for the artist. New York: Watson-Guptill.

Kahrs, J., Calahan, S., Carson, D., \& Poster, S. (1996). Pixel cinematography: A lighting approach for computer graphics (Siggraph '96, Course 30). Available at www.ics.uci.edu/ ses/teaching/ inf290/calahan.pdf.

Koenderink, J. J., \& Pont, S. C. (2003). The secret of velvety skin. Machine Vision \& Applications, 14, 260-268.

Koenderink, J. J., van Doorn, A. J., Kappers, A. M. L., te Pas, S. F., \& PonT, S. C. (2003). Illumination direction from texture shading. Journal of the Optical Society of America A, 20, 987-995.

Koenderink, J. J., van Doorn, A. J., \& Pont, S. C. (2004). Light direction from shad(ow)ed random Gaussian surfaces. Perception, 33, 1405-1420.

Lambert, J. H. (1760). Photometria, sive de Mensura et gradibus luminis, colorum et umbrce. Augsburg: Eberhard Klett.

LANGER, M. S., \& BÜLTHOFF, H. (2001). Human perception of local shape from shading under variable lighting. In CVPR 2001: Proceedings of the 2001 IEEE Computer Society Conference on Computer Vision and Pattern Recognition. Los Alamitos, CA: IEEE Computer Society Press.

Mamassian, P., Knill, D. C., \& Kersten, D. (1998). The perception of cast shadows. Trends in Cognitive Sciences, 2, 288-295.

Mamassian, P., Landy, M., \& Maloney, L. T. (2002). Bayesian modelling of visual perception. In R. P. N. Rao, B. A. Olshausen, \& M. S. Lewicki (Eds.), Probabilistic models of the brain: Perception and neural function (pp. 13-36). Cambridge, MA: MIT Press.

MARR, D. (1982). Vision: A computational investigation into the human representation and processing of visual information. San Francisco: W. H. Freeman.

Moon, P., \& SPEncer, D. E. (1981). The photic field. Cambridge, MA: MIT Press.

Nicodemus, F. E., Richmond, J. C., Hsia, J. J., Ginsberg, I. W., \& LIMPERIS, T. (1977). Geometric considerations and nomenclature for reflectance (Monograph No. 160). Washington, DC: National Bureau of Standards.

Pont, S. C., \& Koenderink, J. J. (2004). Surface illuminance flow. In Y. Aloimonos \& G. Taubin (Eds.), Second International Symposium on 3 D Data Processing, Visualization and Transmission (pp. 2-9). Los Alamitos, CA: IEEE Computer Society Press.

Pont, S. C., \& Koenderink, J. J. (2005). Bidirectional texture contrast function. International Journal of Computer Vision, 62, 17-34.

TE PAS, S. F., \& Pont, S. C. (2005). A comparison of material and illumination discrimination performance for real rough, real smooth and computer generated smooth spheres. In S. N. Spencer (Ed.), Proceedings of the 2nd Symposium on Applied Perception in Graphics and Visualization (pp. 75-81). New York: ACM.

te Pas, S. F., Pont, S. C., \& Paffen, C. L. E. (2004). Consistent errors in human judgments of material and light-source direction. Perception, 33, ECVP Abstract Supplement.

(Manuscript received November 3, 2004; revision accepted for publication July 18, 2006.) 